\title{
Non-rigid Contour-based Registration of Cell Nuclei in 2D Live Cell Microscopy Images Using Dynamic Elasticity Model
}

\author{
Dmitry V. Sorokin, Igor Peterlik, Marco Tektonidis, Karl Rohr, and Pavel Matula
}

\begin{abstract}
The analysis of the pure motion of subnuclear structures without influence of the cell nucleus motion and deformation is essential in live cell imaging. In this work, we propose a 2D contour-based image registration approach for compensation of nucleus motion and deformation. The proposed approach is the extension of our previously published contour-based approach that used an elasticity model to register cell images. Compared to the previous contour-based approach, our approach employs an dynamic elasticity model for forward simulation of nucleus motion and deformation based on the motion of its contours. The contour matching process is embedded as a constraint into the system of equations describing the elastic behavior of the nucleus. This results better performance both in terms of quality of the results and computation time. Our approach was successfully applied to real live cell microscopy image sequences of different types of cells including the data specifically designed to evaluate cell image registration methods. An experimental comparison with existing contour-based registration methods and an intensity-based registration method has been performed. We also studied the dependence of the results on the choice of method parameters.
\end{abstract}

Index Terms-biomedical image analysis, microscopy, image sequence analysis, registration, elasticity, contour-based registration

\section{INTRODUCTION}

$\mathbf{T}$ $\mathrm{HE}$ analysis of subcellular foci motion is one in the most common tasks of live cell imaging. This problem arise in studies related to DNA repair, nucleoli assembly, viral defense and other processes that are important for understanding cell function. However, the motion of foci cannot be sufficiently analyzed directly in raw image sequence as living cells are moving and deforming during imaging process. The observed motion of subcellular foci consists of two components: local motion of the foci and global motion of the nucleus. To get the information about only the subcellular foci motion, the

D.V. Sorokin is with Laboratory of Mathematical Methods of Image Processing, Faculty of Computational Mathematics and Cybernetics, Lomonosov Moscow State University, Leninskie Gory, 2-nd educational building, 119991 Moscow, Russia and Centre for Biomedical Image Analysis, Faculty of Informatics, Masaryk University, Botanická 68a, 60200 Brno, Czech Republic e-mail: sorokin@fi.muni.cz

I. Peterlik is with Institute of Computer Science, Masaryk University, Botanická 68a, 60200 Brno, Czech Republic.

M. Tektonidis and K. Rohr are with University of Heidelberg, BIOQUANT, IPMB, and DKFZ Heidelberg, Dept. Bioinformatics and Functional Genomics, Biomedical Computer Vision Group, Im Neuenheimer Feld 267, 69120 Heidelberg, Germany.

P. Matula is with Centre for Biomedical Image Analysis, Faculty of Informatics, Masaryk University, Botanická 68a, 60200 Brno, Czech Republic

Manuscript received April 19, 2005; revised August 26, 2015. global motion of the nucleus needs to be compensated. This is usually done by means of image registration. Namely all the images in the sequence are normalized to some reference time point (usually the first image of the sequence).

A number of different registration approaches for cell deformation and motion compensation have been presented in previous work. The approaches that use image intensity have been mostly used. In [1] a rigid approach based on phase correlation has been presented. Another correlationbased approach to compute image translations was proposed in [2]. Baheerathan et al. [3] used phase correlation combined with landmark based method to compensate for affine transformation. The approach presented in [4] used inertia tensor computed based on labeled proteins to compensate for nuclei rotation and translation. In [5] the authors used photobleaching model to compensate rigid motion of live cells during FLIP experiments. Raza et al. [6] used a block-matching approach to compensate for translations between color channels in multitag fluorescence microscopy images. Besides rigid methods several non-rigid intensity-based registration approaches were presented. In [7] the authors suggested a parametric motion model for cell motion compensation. In [8] the a semiautomatic landmark-based non-rigid registration approach was proposed. In [9] the authors introduced an intensity-based nonrigid registration approach for compensation of cell nuclei motion and deformation based on the extension of LucasKanade optic flow algorithm. The multi-frame extension of this algorithm was proposed in [10]. In [11] the authors suggested a landmark-based approach for affine registration of moving cells. A few algorithms for registration of 2D slices of 3D static microscopy images were introduced. The algorithms based on using thin-plate splines [12] and B-splines [13], [14] were presented. The method proposed in [15] was based on block-matching algorithm. The authors in [16] presented a feature-based registration approach for $3 \mathrm{D}$ reconstruction of electron microscopy images. In order to register static confocal microscopy images of different objects an approach based on B-splines was presented in [17] and a landmark-based algorithm that use thin-plate splines was proposed in [18].

Intensity-based registration approaches demonstrate high efficiency due to the fact that they use all the information presented in the image sequence. However these methods are not very suitable for image data where the image intensities are not very reliable to be used for image registration (e.g., high level of noise, large and abrupt motion of cellular structures). For example, the methods based on optic flow principles [9], 


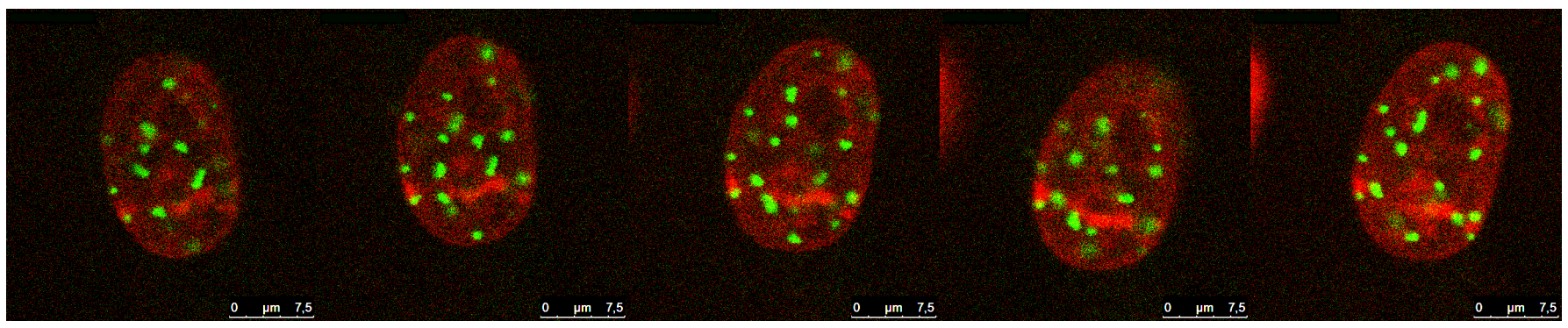

(a) Two-channel data. Structures of interest presented in green channel. Nucleus presented in red channel.

Fig. 1. Example images from the time-lapse image sequences (contrast-enhanced).

[10] or block-matching [15] require image intensities to be stable which can not be fulfilled in case of strong intensity changes (e.g. due to photobleaching and noise). In [7] a solution to intensity variation problem was suggested however the proposed registration approach employed affine deformation model which cannot cope with strong local deformations. The landmark-based algorithms [8], [11]-[13], [16], [18] require stable landmarks to be localized in the registered pair of images which can be difficult as often there is no a priori biologically stable structures in the moving cell. Also, when the subject of the study is the local motion of subcellular structures the intensity-based registration algorithms can influence the motion patterns of these structures while compensating the cell motion and deformation. Thus the errors can be introduced into the trajectories of the structures of interest. This problem can be solved by acquiring the images in 2 channels [9], [10] where the objects of interest are represented in one channel and some stable structure (e.g. chromatin) is represented in the second channel. The approaches [9], [10] were applied to register the nucleus channel of two-channel microscopy image sequences and the computed transformations could be used to compensate the nucleus motion and deformation in the particle channel. However it is not always possible to acquire multichannel images due to different biological experimental setups. The mentioned limitations of intensitybased registration approaches led to the need of development of cell motion compensation approaches that do not use image intensity for registration.

In previous work on registration of cell images in microscopy data, only a few approaches that do not use image intensity have been presented. In [19] the authors used a modelbased approach where yeast cells were fitted with an ellipses to compensate for rigid motion in 2D image sequences. Matula et al. [20] introduced a point-based rigid registration approach where the positions of subcellular structures were used to find the suitable transformation between the cell in different frames. A shape based approach using distance functions was introduced in [21]. Several non-rigid registration approaches without using image intensity were proposed. In [22] the authors used an extension of demons algorithm applied to cell segmentation masks to compensate for global motion and deformation of living cells. De Vylder et al. [23] introduced a contour-based non-rigid registration method for dynamic cell nuclei where the deformation is defined based on the correspondences between the contour points of the nucleus in consecutive time frames.

The described approaches do not use image intensity but binary segmentations of the nucleus. The efficiency of the methods were demonstrated on both real microscopy data and synthetically created cell phantom images. These approaches were mostly applied to the data acquired in one channel. However in [19], [20] the algorithms could compensate only the rigid motion of the cell. In [21], [22] the authors described non-rigid approaches but the implied deformation models allow to compensate only limited deformations that is not very effective in the case of abrupt motion. In [23] the approach was specifically designed to cope with strong deformations however due to the uniform sampling of the nucleus boundary the contour matching algorithm introduces errors in the deformation fields. In addition, the thin-plate deformation model used in [23] is a relatively rough approximation of the nucleus deformation. In [24] the authors introduced a cell nucleus shape normalization approach based on linear elasticity. However the approach [24] was applied to static cell nuclei to normalize their shape to 3D sphere and measure relative position of internal structures.

In this contribution, we propose a new contour-based nonrigid registration approach. In our approach we use dynamic non-linear elasticity model to compensate for cell motion and deformation. This work extends our previous work [25] where elastostatics model employing Navier equation with Dirichlet boundary conditions was used to register the cell nuclei. The method described in [25] employed "global-tolocal" registration strategy that required two steps to be performed: the compensation for nucleus motion by rigid alignment of the images and the compensation for nucleus deformation by solving the Navier equation. The registration was performed for each pair of two consecutive frames resulting the backward deformation field from frame $k$ to frame $k-1$. The Navier equation was solved using the Dirichlet boundary conditions defined by the contour matching of two consecutive frames. As distinct from the previous approach [25], the method presented in this contribution is based on the forward simulation of the elastic motion of the nucleus resulting the full model of nucleus deformation given on triangulation that can be used to construct both forward and backward deformation fields for cell image registration. The proposed approach does not require rigid alignment step as the new contour matching procedure is capable to compensate for large rigid displacements. The contour matching is embedded 


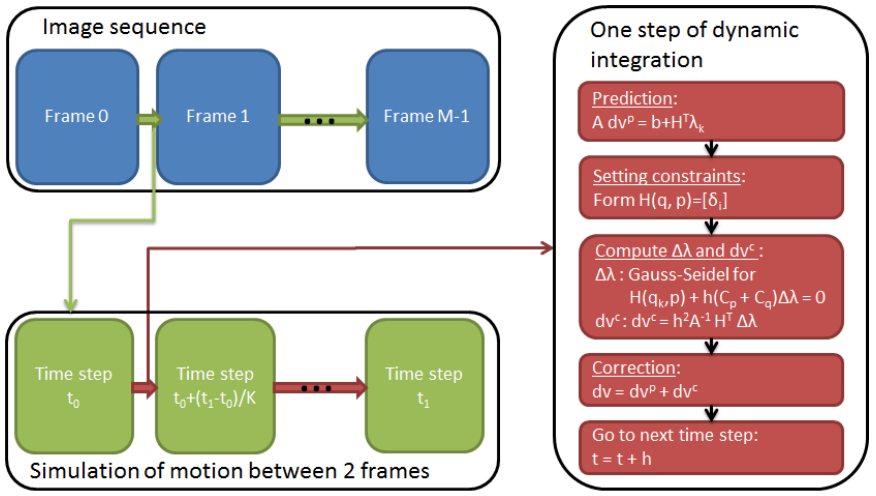

Fig. 2. Forward nucleus motion modeling scheme.

as constraints into the system of equations of the elastic motion model. The system of equations is iteratively solved using prediction-correction scheme. We also significantly extended the quantitative evaluation of the proposed approach. The method was evaluated on real fluorescent microscopy image data including the data specifically designed and acquired for testing cell image registration techniques [26]. The approach was compared with the method described in our previous work [25], the contour-based method presented in [23] and the intensity-based method [9].

The paper is organized as follows. In the next section we introduce our contour-based non-rigid registration approach. Then we present the evaluation results and method parameter study. In the final sections of the paper we conclude the paper with a discussion.

\section{Methods}

In this section, we describe our contour-based registration method. Prior to registration we perform threshold-based segmentation and determine the contours of cell nuclei. Below, we first describe the data representation and overall scheme of our registration approach (see Fig.2). Then, we explain the physical model used for the registration and its difference from our previous approach [25]. After that we detail the main parts of the approach, namely the simulation of nucleus motion, the definition of contour matching constraints, and the numerical solution scheme.

\section{A. Data Representation and Forward Nucleus Motion Simu- lation}

To perform the registration of every image of the sequence to the first image we use the forward simulation of nucleus motion based on the model described in the next subsection based on finite elements (FE) method. Given the binary images of cell nucleus in every frame we define nucleus contours $\mathcal{C}_{k}$ where $k=0, \ldots, M-1$ and $M$ is the number of images in the sequence. Each point of the contour is represented by its position $P$ and outer normal $\mathbf{n}_{P}$. Then we define the triangulation $\mathcal{T}_{0}$ with $N$ nodes for the first frame of the sequence where $\mathcal{E}_{0}$ is the set of triangulation elements and $\mathcal{N}_{0}$ is the set of nodes. The idea of forward simulation of nucleus motion is to simulate the elastic motion of the nucleus given only the contours $\mathcal{C}_{k}$. In order to do this, the triangulation $\mathcal{T}_{0}$ is iteratively deformed based on the model described below so that its boundary optimally fits the contour $\mathcal{C}_{1}$. Let $\mathcal{T}_{1}$ be the result of this operation representing the deformed nucleus in the second frame of the sequence. The same operation is performed consequently for $\mathcal{T}_{k}$ and $\mathcal{C}_{k+1}$ resulting the set of triangulations $\mathcal{T}_{k}(k=1, \ldots, M-1)$ that represent the deformed nucleus in the corresponding frame.

Given the triangulation representing the deformed nucleus in every frame $\mathcal{T}_{k}(k=0, \ldots, M-1)$ one can compute the forward deformation field $\mathbf{u}_{0 \rightarrow k}=\mathbf{q}^{k}-\mathbf{q}^{0}$ or backward deformation field $\mathbf{u}_{0 \leftarrow k}=\mathbf{q}^{0}-\mathbf{q}^{k}$ where $\mathbf{q}^{0}, \mathbf{q}^{k} \in \mathbb{R}^{2 N}$ are the vectors that consist of $\mathcal{T}_{0}$ and $\mathcal{T}_{k}$ triangulations node positions ( $\mathbf{q}=$ $\left.\left[x_{0}, y_{0}, x_{1}, y_{1}, \ldots, x_{N-1}, y_{N-1}\right]^{T}\right)$. Thus $\mathbf{u}_{0 \rightarrow k}$ and $\mathbf{u}_{0 \leftarrow k}$ are defined in the nodes of triangulation. The forward deformation fields $\mathbf{u}_{0 \rightarrow k}$ can be interpolated in every pixel of the image and used to wrap the image sequence to normalize it to the first frame of the sequence. The backward deformation field $\mathbf{u}_{0 \leftarrow k}$ can be used to register a set of features (e.g. the position of foci the local motion of which is studied).

\section{B. Modeling the Nucleus Deformation Based on Linearized Elasticity and Dynamic Integration}

As it was shown in [27] cell nucleus demonstrates elastic behavior. Thus, the deformation of the nucleus can be modeled using elasticity theory [28] considering cell nucleus an isotropic homogenous material.

The nucleus motion can be described using the second Newton's law

$$
\begin{aligned}
& M \mathbf{a}=\mathbf{f}_{i n t}+\mathbf{f}, \\
& \mathbf{f}_{i n t}=\mu \Delta \mathbf{u}+(\mu+\lambda) \nabla(\nabla \cdot \mathbf{u}),
\end{aligned}
$$

where $M$ is the nucleus mass, a is the acceleration, $\mathbf{u}$ represents the displacement and $\mathbf{f}$ represents the external forces applied to the nucleus boundary due to constrains that drive the registration. The Lamé coefficients $\mu$ and $\lambda$ define the properties of the elastic material and connected to the Poisson's ratio and Young's modulus with the following relations

$$
\lambda=\frac{\nu E}{(1+\nu)(1-2 \nu)}, \mu=\frac{E}{2(1+\nu)} .
$$

The $\mathbf{f}_{i n t}$ term represents the internal elastic forces that present in the nucleus. In [25] we used the elastostatic approach exploiting Navier-Cauchy equation to model the nucleus motion:

$$
\mathbf{f}_{i n t}=0,
$$

where $\mathbf{f}_{i n t}$ is defined in (1) and the contour matching constrains that drove the deformation were defined as Dirichlet boundary conditions. In this contribution we employ the dynamic model by adding the $M$ a term responsible for the nucleus inertia. The contour matching constrains that drive the deformation are defined using the external forces $\mathbf{f}$.

In order to solve (1) we employ FE method. Let us consider the nucleus triangulation $\mathcal{T}_{k}$ in frame $k$ and the target contour of the nucleus $\mathcal{C}_{k+1}$ in frame $k+1$. Then the motion of the nucleus can be presented by the Newton's second law rewritten in the following form 


$$
\begin{aligned}
& \mathbf{M} \ddot{\mathbf{q}}=\mathbb{F}(\mathbf{q}, \dot{\mathbf{q}}), \\
& \mathbb{H}(\mathbf{q}, \mathbf{p})=0,
\end{aligned}
$$

where $\mathbf{q} \in \mathbb{R}^{2 N}$ is the vector of triangulation node positions, $\mathbf{p} \in \mathbb{R}^{2 N_{c}}$ is the vector of points in the target contour $\mathcal{C}_{k+1}$ $\left(N_{c}\right.$ is the number of points in $\left.\mathcal{C}_{k+1}\right), \mathbf{M} \in \mathbb{R}^{2 N \times 2 N}$ is the mass matrix (the nucleus is considered uniform and thus the mass matrix does not depend on q). The internal elastic forces component $\mathbf{f}_{i n t}$ in (1) is represented with the mapping $\mathbb{F}(\mathbf{q}, \dot{\mathbf{q}}): \mathbb{R}^{2 N} \rightarrow \mathbb{R}^{2 N}$. The constraints given by the external forces $\mathbf{f}$ in (1) on the boundary edges of $\mathcal{T}_{k}$ are given in the implicit form using the mapping $\mathbb{H}(\mathbf{q}, \mathbf{p}): \mathbb{R}^{2 N_{c}} \rightarrow \mathbb{R}^{2 N_{c}}$. The problem (2) is considered as a constrained optimization problem. We use the Lagrange multipliers method to solve it

$$
\mathbf{M} \ddot{\mathbf{q}}=\mathbb{F}(\mathbf{q}, \dot{\mathbf{q}})+\mathbf{H}^{T}(\mathbf{q}) \lambda,
$$

where $\mathbf{H}(\mathbf{q})=\frac{\partial \mathbb{H}}{\partial \mathbf{q}}$ is the Jacobian of the constraint mapping $\mathbb{H}(\mathbf{q}, \mathbf{p})$ and $\lambda \in \mathbb{R}^{2 N_{c}}$ is the corresponding vector of Lagrange multipliers. Thus $\mathbf{H}^{T}(\mathbf{q})$ defines the direction of constraint forces and $\lambda$ represents the constraint force amplitudes (the details of constraints definition is described in section II-C).

Let us consider the time interval $\left[t_{k}, t_{k}+h\right]$. Integrating (3) with respect to time $t$ we get

$$
\begin{aligned}
& \mathbf{M}\left(\dot{\mathbf{q}}_{k+h}-\dot{\mathbf{q}}_{k}\right)=\int_{t_{k}}^{t_{k}+h} \mathbb{F}(\mathbf{q}, \dot{\mathbf{q}}) d t+h \mathbf{H}^{T}(\mathbf{q}) \lambda, \\
& \mathbf{q}_{k+h}=\mathbf{q}_{k}+\int_{t_{k}}^{t_{k}+h} \dot{\mathbf{q}} d t .
\end{aligned}
$$

We use the implicit Euler method to compute the integrals in the right part of Eq. (4):

$$
\begin{aligned}
& \mathbf{M}\left(\dot{\mathbf{q}}_{k+h}-\dot{\mathbf{q}}_{k}\right)=h \mathbb{F}\left(\mathbf{q}_{k+h}, \dot{\mathbf{q}}_{k+h}\right)+h \mathbf{H}^{T}\left(\mathbf{q}_{k+h}\right) \lambda_{k+h}, \\
& \mathbf{q}_{k+h}=\mathbf{q}_{k}+h \dot{\mathbf{q}}_{k+h} .
\end{aligned}
$$

As it is described in section II-B we assume that the deformations are small thus we can linearize $\mathbb{F}$ using Taylor expansion:

$$
\mathbb{F}\left(\mathbf{q}_{k}+d \mathbf{q}, \dot{\mathbf{q}}_{k}+d \dot{\mathbf{q}}\right)=\mathbf{f}_{k}+\frac{\delta \mathbb{F}}{\delta \mathbf{q}} d \mathbf{q}+\frac{\delta \mathbb{F}}{\delta \dot{\mathbf{q}}} d \dot{\mathbf{q}} .
$$

Let us denote $\mathbf{K}=\frac{\delta \mathbb{F}}{\delta \mathbf{q}}$ and $\mathbf{B}=\frac{\delta \mathbb{F}}{\delta \dot{\mathbf{q}}}$. These matrices are known as the stiffness and damping matrices. The details on $\mathbf{K}$ and $\mathbf{B}$ matrices construction can be found in [29]. In order to compensate for effects appearing in the case of large displacements the corrotational formulation for stiffness matrix construction is used [30]. The explicit form of these matrices is given in Appendix A. Substituting (6) into (5) and using $d \mathbf{q}=\mathbf{q}_{k+h}-\mathbf{q}_{k}=h \dot{\mathbf{q}}_{k+h}$ and $d \dot{\mathbf{q}}=\dot{\mathbf{q}}_{k+h}-\dot{\mathbf{q}}_{k}$ we obtain

$$
\underbrace{\left(\mathbf{M}+h \mathbf{B}+h^{2} \mathbf{K}\right)}_{\mathbf{A}} d \dot{\mathbf{q}}=\underbrace{-h^{2} \mathbf{K} \dot{\mathbf{q}}_{k}}_{\mathbf{b}}+h \mathbf{H}^{T}\left(\mathbf{q}_{k+h}\right) \lambda_{k+h} .
$$

This equation defines the change in velocity in the nodes of the nucleus triangulation during one time step. The method

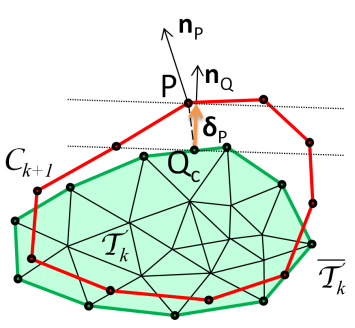

(a)

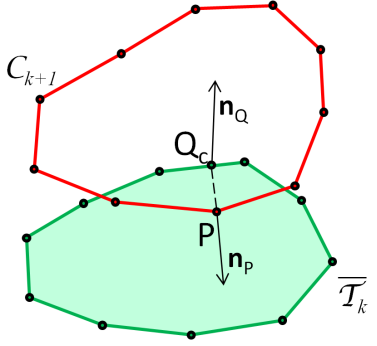

(b)
Fig. 3. (a) Constrain forces definition scheme. The green polygon line represents the nucleus contour $\overline{\mathcal{T}}_{k}$ in frame $k$. The red polygon line represents the target nucleus contour $\mathcal{C}_{k+1}$ in frame $k+1$. The orange arrow represents the constraint force $\delta_{P}$ applied to the point $Q_{c}$. Black dotted lines represent the tangent of $\overline{\mathcal{T}}_{k}$ in point $Q_{c}$ and a parallel to this tangent in the point $P$. (b). The case when $\mathbf{n}_{p} \cdot \mathbf{n}_{q}<0$.

parameters that control the elastic properties of the nucleus, namely Young's modulus $E$ and Poisson's ratio $\nu$, are contained in the matrix $\mathbf{K}$. The only unknown term in this equation is $\mathbf{H}\left(\mathbf{q}_{k+h}\right) \lambda_{k+h}$ the computation of which is discussed in the next sections.

\section{Definition of Contour Matching Constraints}

Let us consider the nucleus triangulation $\mathcal{T}_{k}$ in frame $k$ and target contour of the nucleus $\mathcal{C}_{k+1}$ in frame $k+1$. Let us also denote the subset of edges $\overline{\mathcal{T}}_{k}$ that connects the boundary points of the triangulation $\mathcal{T}_{k}$ (see Fig. 3a). For every point $P \in \mathcal{C}_{k+1}$ we find the closest point $Q_{c} \in \overline{\mathcal{T}}_{k}$ that $\mathbf{n}_{P} \cdot \mathbf{n}_{Q}>0$ where $\mathbf{n}_{P}$ and $\mathbf{n}_{Q}$ are the normals in points $P$ and $Q_{c}\left(Q_{c}\right.$ and $\mathbf{n}_{Q}$ are calculated as linear interpolation of neighboring nodes of $\overline{\mathcal{T}}_{k}$ ). The latter condition is necessary to avoid points $Q_{c}$ located on the opposite side of the nucleus in the case of large frame displacements between frames (see Fig. 3b). Then we define the gap between $P$ and $Q_{c}$ as

$$
\delta_{P}=\left(P-Q_{c}\right) \cdot \mathbf{n}_{Q} .
$$

This gap is zero when the point $P$ is located on the tangent of $\overline{\mathcal{T}}_{k}$ in the point $Q_{c}$.

Such gaps are defined for all $P_{i} \in \overline{\mathcal{T}}_{k}, i=\overline{0, N_{c}-1}$. The corresponding points $Q_{c i} \in \overline{\mathcal{T}}_{k}$ are obtained using the ICP algorithm [31]. We consider the contours to be matched when $\delta_{P_{i}}=0$ for $\forall i=\overline{0, N_{c}-1}$. Thus the constraint mapping $\mathbb{H}(\mathbf{q}, \mathbf{p})$ can be defined as

$$
\mathbb{H}(\mathbf{q}, \mathbf{p})=\left[\begin{array}{c}
\delta_{P_{0}} \\
\vdots \\
\delta_{P_{N_{c}-1}}
\end{array}\right], P_{i} \in \mathcal{C}_{k+1} .
$$

It is important to note that the points $Q_{c i} \in \overline{\mathcal{T}}_{k}$ are the points of the same elastic body. Thus applying the constraint in one point influence the other points. So the optimal minimization of the gaps (i.e. contour matching) taking into account the elasticity of the body is obtained using the Lagrange multipliers $\lambda_{k+h}$ in (7). To define $\lambda_{k+h}$ and solve (7) we use a prediction-correction solution scheme which is described in the next subsection. 


\section{Prediction-Correction Solution Scheme}

In this section we describe the computation scheme allowing to solve the equation (7). First, to simplify the solution process we assume that the constraint Jacobian $\mathbf{H}^{T}(\mathbf{q})$ does not change during constraints resolving thus instead of $\mathbf{H}^{T}\left(\mathbf{q}_{k+h}\right)$ we use $\mathbf{H}^{T}\left(\mathbf{q}_{k}\right)=\mathbf{H}^{T}$. In the following, we use the notation $\mathbf{H}$ to emphasize that this matrix does not change during time step. Then, substituting this into (7) we obtain

$$
\mathbf{A} d \dot{\mathbf{q}}=\mathbf{b}+h \mathbf{H}^{T} \lambda_{k+h} .
$$

The solution consists of three steps: prediction, constraint solving and correction. Let us describe these steps.

Step 1. First we solve (9) setting $\lambda_{k+h}=0$

$$
\mathbf{A} d \dot{\mathbf{q}}^{p}=\mathbf{b} .
$$

Thus we obtain the predictive change in velocity $d \dot{\mathbf{q}}^{p}$ that corresponds to the unconstrained motion that continue from the previous step (analogous to body inertia).

Step 2. On this step we resolve the constraints and get the correction for the vector of Lagrange multipliers $\lambda_{k+h}$. Assuming $\mathbf{b}=0$ we get

$$
\mathbf{A} d \dot{\mathbf{q}}^{c}=h \mathbf{H}^{T} \lambda_{k+h} .
$$

In (11) the correction change in velocity $d \dot{\mathbf{q}}^{c}$ and the vector of Lagrange multipliers $\lambda_{k+h}$ are unknown. To obtain the relation between these variables we linearize the constraints equation with respect to velocity change:

$$
\mathbb{H}\left(\mathbf{q}_{k+h}, \mathbf{p}\right)=\mathbb{H}\left(\mathbf{q}_{k}, \mathbf{p}\right)+h \mathbf{H}^{T} d \dot{\mathbf{q}}^{c}
$$

According to (2) the constraints are satisfied when $\mathbb{H}\left(\mathbf{q}_{k+h}, \mathbf{p}\right)=0$. Taking this into account and substituting the solution of (11) into (12) we get the following non-linear equation

$$
\mathbb{H}\left(\mathbf{q}_{k}, \mathbf{p}\right)+h \mathbf{H} \mathbf{A}^{-1} \mathbf{H}^{T} \lambda_{k+h}=0 .
$$

In this equation the term $\mathbf{C}_{q}=\mathbf{H} \mathbf{A}^{-1} \mathbf{H}^{T}$ called source object compliance matrix is responsible for the elastic behavior of the nucleus in the constraints solution. In (13) any physical properties of the target object represented by $\mathcal{C}_{k+1}$ are not taken into account as this object is a purely geometrical entity in this simulation. Although the solution of (13) can suffer from inaccuracies in extraction of $\mathcal{C}_{k+1}$ from the source images. To increase the numerical stability of the solution process we introduce to (13) the target object compliance matrix $\mathbf{C}_{p}$ similar to [32], [33]. However since the object $\mathcal{C}_{k+1}$ does not have any physical properties, the matrix $\mathbf{C}_{p}$ is diagonal: $\mathbf{C}_{p}=\gamma \mathbf{I}_{2 N_{c}}$. The additional method parameter $\gamma$ is called target compliance. Thus we obtain the following equation

$$
\mathbb{H}\left(\mathbf{q}_{k}, \mathbf{p}\right)+h\left(\mathbf{C}_{q}+\mathbf{C}_{p}\right) \lambda_{k+h}=0 .
$$

which is solved by Gauss-Seidel method with respect to $\lambda_{k+h}$.
Step 3. After we obtain the values of $\lambda_{k+h}$ we can compute the correction change in velocity $d \dot{\mathbf{q}}^{c}$ using (11). We compute the overall change in velocity during time step as

$$
d \dot{\mathbf{q}}=d \dot{\mathbf{q}}^{p}+d \dot{\mathbf{q}}^{c}=\mathbf{A}^{-1} \mathbf{b}+h^{2} \mathbf{A}^{-1} \mathbf{H}^{T} \lambda_{k+h} .
$$

Then we can obtain the velocity on the next time step as $\dot{\mathbf{q}}_{k+h}=\dot{\mathbf{q}}_{k}+d \dot{\mathbf{q}}$ and subsequently the triangulation nodes positions on the next time step $\mathbf{q}_{k+h}=\mathbf{q}_{k}+h \dot{\mathbf{q}}_{k+h}$. Thus the problem (5) is solved and we can proceed to the next time step.

The iterative solution process is continued until the equilibrium is reached i.e. the triangulation $\mathcal{T}_{k}$ representing the nucleus in frame $k$ fits the contour $\mathcal{C}_{k+1}$. Typically 20 time steps is enough to reach the equilibrium. Then we proceed to the next pair of frames taking the deformed triangulation as the input. The overall scheme of the simulation process is shown in Fig. 2.

\section{EXPERIMENTAL RESULTS}

For quantitative evaluation of our approach we used two datasets of real microscopy image sequences of live cells. The image sequences in both datasets contained the structures that are stable w.r.t. the nucleus (i.e., there is no relative motion). These stable structures were used to assess the registration error of the proposed algorithm in comparison to the contourbased approaches from [23], [25] and intensity-based approach from [9]. The images from the first dataset contain local stable features manually tracked by human annotators that allowed to study the methods in case of significant rotation, translation and shrinkage of the cell. The images from the second dataset contain automatically tracked features that occupy significant part of the nucleus and enable to compare the methods efficiency in the interior part of the nucleus and on its periphery. The sequences from the second dataset also have strong intensity variations and nucleus deformation which allows to test the methods in the case of intensity changes.

\section{A. Evaluation Datasets Description}

The first dataset consists of three image sequences that were used for the evaluation of the approach in [25]. In these image sequences the stable structures were manually tracked by annotators. In two sequences (denoted as Seq. A1 and Seq. A2) the nucleus of U2OS cell was UV-irradiated in a stripe-like region. The UV-irradiated stripe is visible in the nucleus channel and stable w.r.t. the nucleus. In both sequences keypoints of the UV-irradiated stripe were manually tracked by three independent annotators. The nuclei in these image sequences undergo significant translation (including jumps) and rotation along with nucleus deformations. In addition, the image noise is relatively high.

The third image sequence (denoted as Seq. A3) depicts U2OS cell nucleus going into mitosis. For the evaluation 9 spot-like structures in the nucleus channel are used as keypoints that were manually tracked by one annotator. The structures are stable w.r.t. the nucleus and represent the motion and deformation of the nucleus. 
TABLE

EVALUATION SEQUENCES DESCRIPTION

\begin{tabular}{cccc}
\hline Image sequence & Frame size & Number of frames & Features \\
\hline A1 & $512 \times 512$ & 25 & 4 keypoints \\
A2 & $512 \times 512$ & 38 & 5 keypoints \\
A3 & $512 \times 512$ & 66 & 9 keypoints \\
\hline B1 & $287 \times 356$ & 42 & 4 line-features, \\
B2 & $512 \times 512$ & 30 & 4 inner points, \\
B3 & $279 \times 318$ & 42 & 8 end points \\
B4 & $322 \times 304$ & 35 & \\
\hline
\end{tabular}

TABLE II

REGISTRATION ERROR FOR DIFFERENT APPROACHES APPLIED FOR IMAGE SEQUENCES FROM THE FIRST EVALUATION DATASET.

\begin{tabular}{l||cccc|cccc|c}
\multicolumn{8}{c|}{ Mean error $\bar{e}_{\text {mean }}$ [pixels] } \\
\begin{tabular}{l||cccc|ccccc|c} 
Sequence \\
Annotator
\end{tabular} & $A_{1}$ & $A_{2}$ & $A_{3}$ & Avg. & $A_{1}$ & $A_{2}$ & $A_{3}$ & Avg. & \\
\hline Unregistered & 30.17 & 30.75 & 31.29 & 30.74 & 49.42 & 50.20 & 49.05 & 49.55 & 26.65 \\
Cont.-based [23] & 8.72 & 10.42 & 9.47 & 9.53 & 18.87 & 19.64 & 20.20 & 19.57 & 9.96 \\
Int. pairwise [9] & 7.78 & 8.79 & 7.21 & 7.92 & 9.07 & 9.84 & 9.47 & 9.46 & $\mathbf{3 . 0 7}$ \\
Cont.-based [25] & 5.96 & 7.20 & 6.01 & 6.39 & 8.23 & 9.45 & 8.97 & 8.89 & 7.43 \\
Proposed & $\mathbf{5 . 7 1}$ & $\mathbf{7 . 1 8}$ & $\mathbf{5 . 7 8}$ & $\mathbf{6 . 2 2}$ & $\mathbf{7 . 9 5}$ & $\mathbf{9 . 1 0}$ & $\mathbf{8 . 7 8}$ & $\mathbf{8 . 6 1}$ & 6.73
\end{tabular}

For Seq. A1 and Seq. A2 the ground truth was obtained by manual tracking by three different annotators to increase the reliability of the evaluation, since the data is quite noisy and the structures are rather blured. In Seq. A3 the spot-like structures are better visible and thus could be tracked reliably by one annotator.

The second dataset contains four image sequences (denoted as Seq. B1-B4) obtained using procedure described in [26]. These sequences depict HeLa cells with histone H2B tagged by GFP. In each sequence four regions of interest were bleached using argon laser. The bleached structures represent a grid of four orthogonally crossing lines (see Fig. 4). These bleached structures are stable w.r.t. the nucleus and represent the motion and deformation of the nucleus [26]. In all the sequences the bleached structures were automatically detected using the algorithm described in [34] resulting line features that were used for the evaluation of the registration approach. In addition, we studied the behavior of the registration approaches on the periphery of the nucleus using the end points of the line features (further referred as end points), and on the interior part of the nucleus using the intersections of the line features (further referred as inner points, see Fig. 4).

The details of the image sequences A1-A3, B1-B4 are given in Table I.

\section{B. Evaluation Results}

For each keypoint in Seq. A1-A3 we computed the registration error as the Euclidean distance to the position of the keypoint in the first image:

$$
e_{i}^{k}=\left\|\mathbf{p}_{i}^{k}-\mathbf{p}_{i}^{1}\right\|
$$

where $\mathbf{p}_{i}^{k}$ is the coordinate of the $\mathrm{i}$-th feature point in the $\mathrm{k}$-th frame. The same registration error was computed for the inner points and end points in Seq. B1-B4.

For line-features in Seq. B1-B4 we computed the registration error for every frame as the Fréchet distance to the line-features in the first frame.
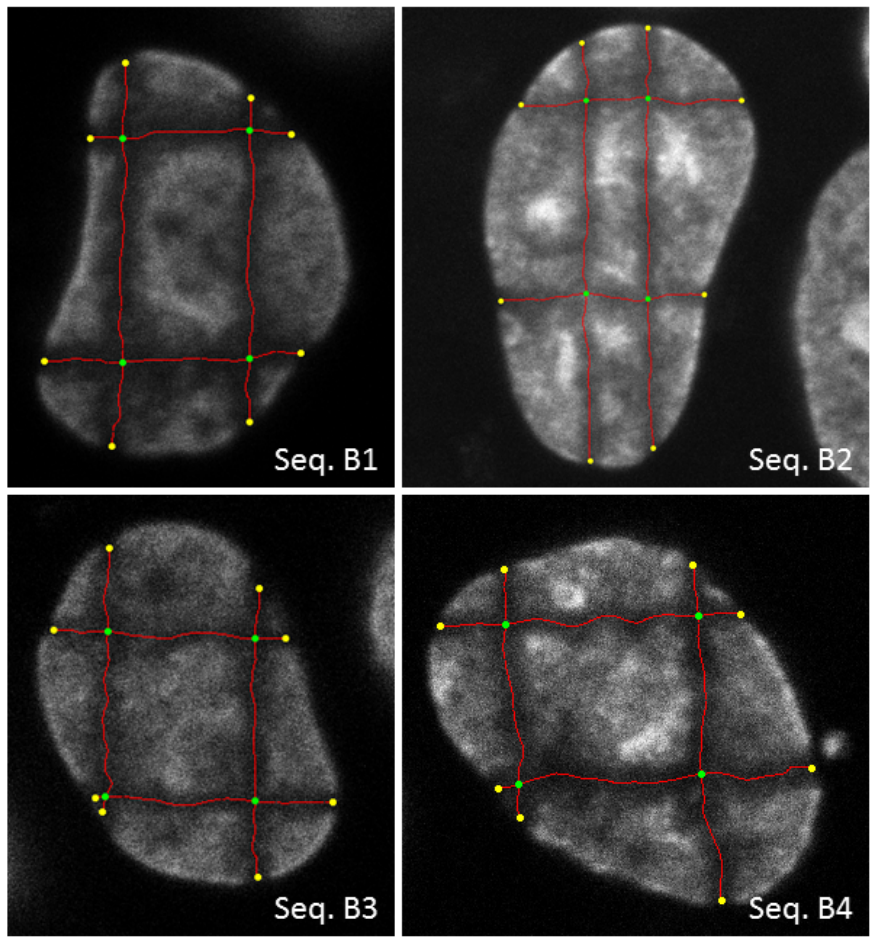

Fig. 4. The first image of the image sequences B1-B4 from the second evaluation detaset. The red lines represent the line features, the green dots represent the inner points, and the yellow dots represent the end points. The images are cropped and scaled to fit the figure.

The parameters for the tested approaches were set to the optimal values to suit our data. For the contour-based approach [23] we used 100 contour sampling points and thin-plate splines for the deformation field interpolation. For contour matching we used the normalized centroid distance signature in Seq. A1, A2, B2, the curvature signature in Seq. A3, B1 and the tangent direction signature in Seq. B3, B4. For the intensity-based approach [9] we used symmetric weighting scheme and $\sigma=2$ for regularization of the deformation and update field. For both elasticity-based approaches we used the parameter values according to the real properties of cell nucleus. The Poisson's ratio of the nucleus was set to 0.4 [35]. The Young's modulus of the nucleus was set to 10 $\mathrm{kPa}$ [36] for Seq. A1-A2 and Seq. B1-B4. For Seq. A3 we set the Young's modulus to $2 \mathrm{kPa}$ as the Young's modulus of the nucleus in mitosis was reported to drop 5 times [37]. The triangulation for the FE method in both elasticitybased approaches was constructed based on nucleus contours represented by 100 sampling points (to be consistent with the number of contour points used in contour-based approach [23]). The target compliance for the proposed approach was set to $\gamma=0.5$.

The registration error $\bar{e}_{\text {mean }}$ averaged over all time points and all keypoints is shown in Table II for Seq. A1 and Seq. A2 for the three different annotators. In addition, the average values over the three annotators has been included. It can be seen that the variation of $\bar{e}_{\text {mean }}$ for the different annotators for the unregistered case is rather low which indicates the consistency of the selected keypoints. The average values for $\bar{e}_{\text {mean }}$ 
TABLE III

REGISTRATION ERROR FOR LINE FEATURES, INNER POINTS AND END POINTS FOR DIFFERENT APPROACHES APPLIED FOR IMAGE SEQUENCES FROM THE SECOND EVALUATION DATASET.

\begin{tabular}{l||cccc|cccc|cccc}
\multicolumn{1}{l|}{} & \multicolumn{4}{c}{ Line features } & \multicolumn{4}{c}{ Inner points } & \multicolumn{4}{c}{ End points } \\
Sequence & B1 & B2 & B3 & B4 & B1 & B2 & B3 & B4 & B1 & B2 & B3 & B4 \\
\hline Unregistered & 22.91 & 11.54 & 12.43 & 11.92 & 11.30 & 5.71 & 8.00 & 6.79 & 18.15 & 6.40 & 9.30 & 8.13 \\
Cont.-based [23] & 24.35 & 9.13 & 9.02 & 11.58 & 14.60 & 5.50 & 5.33 & 4.57 & 15.94 & 3.44 & 4.92 & 7.63 \\
Int.-based [9] & 25.99 & 11.16 & 9.28 & 9.99 & 22.47 & 7.50 & 6.56 & 4.43 & 9.42 & 4.56 & 5.01 & 2.51 \\
Cont.-based [25] & 12.79 & 8.17 & 5.83 & 7.25 & 10.69 & 5.45 & 3.17 & 3.46 & 7.93 & 2.60 & 2.98 & $\mathbf{2 . 0 1}$ \\
Proposed & $\mathbf{9 . 7 2}$ & $\mathbf{7 . 8 2}$ & $\mathbf{5 . 3 2}$ & $\mathbf{6 . 9 3}$ & $\mathbf{7 . 9 2}$ & $\mathbf{5 . 2 2}$ & $\mathbf{2 . 7 6}$ & $\mathbf{3 . 0 4}$ & $\mathbf{3 . 6 9}$ & $\mathbf{2 . 0 2}$ & $\mathbf{2 . 1 7}$ & 2.43
\end{tabular}

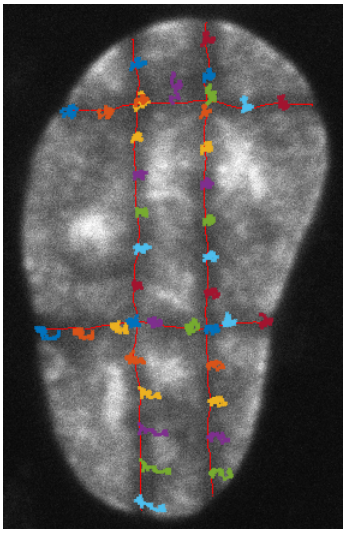

(a)

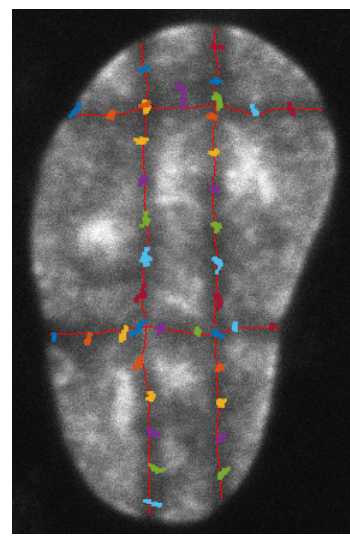

(b)

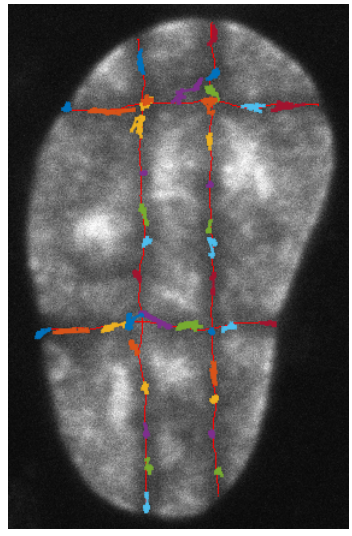

(c)

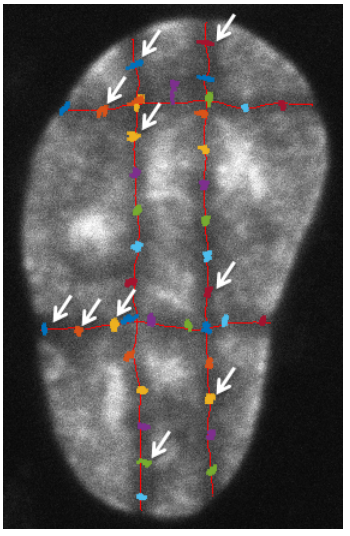

(d)

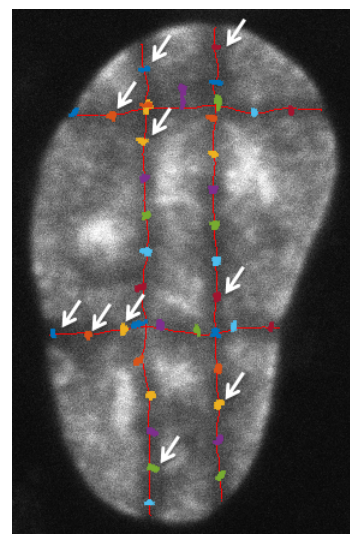

(e)

Fig. 5. Tracks of line features of Seq. B2 overlaid with the first image of the sequence. The tracks of the line features are represented as the tracks of the points of the line features sampled with 30 pixel interval for better visibility. The tracks of line features are shown for (a) unregistered data, (b) after registration performed with the contour-based approach [23], (c) after registration performed with the intensity-based approach [9], (d) after registration performed with the contour-based approach [25], and (e) after registration performed with the proposed approach. White arrows indicate the tracks with the most visible difference between (d) and (e).

over the three annotators show that the proposed approach outperforms the previous contour-based approaches [23] and [25] by $53 \%$ and $3 \%$ respectively for Seq. A1, and by $127 \%$ and 3\% respectively for Seq. A2. Compared to the intensity-based approach [9] the proposed approach yields a $27 \%$ improvement for Seq. A1 and 10\% improvement for Seq. A2 while the intensity-based approach exploits more information using the image intensities. The registration error $\bar{e}_{\text {mean }}$ for Seq. A3 is also shown in Table II. For Seq. A3 the result of the proposed approach is better compared to the previously introduced contour-based approaches by $48 \%$ and $10 \%$ respectively but worse compared to the intensity-based approach. The reason is that the intensity-based approach exploits the intensity information within the nucleus (which is going into mitosis) while the contour-based approaches do not depend on the intensity variation which can be an advantage for certain data.

The registration error $\bar{e}_{\text {mean }}$ averaged over all time points and all line features for Seq. B1-B4 is shown in Table III. The proposed approach gives better results than both previously introduced contour-based approaches for all the sequences from the second dataset. Namely, the proposed approach yields in average $76 \%$ improvement in comparison to the contourbased approach [23] and 13\% improvement in comparison to the contour-based approach [25]. In addition, the proposed approach outperforms (in average by $82 \%$ ) the intensitybased approach for all sequences in the second dataset. The reason for low performance of the intensity-based method for the second dataset is that there is a substantial change in nucleus intensity throughout the sequences caused by imaging conditions. While the method [9] is based on the extension of Lucas-Kanade optic flow algorithm which requires the image intensity to be stable.

We also analyzed the behavior of the proposed approach in the central part of the nucleus and its periphery by using the inner points and the end points of the line features. The comparison of the registration error averaged over all time points for end points and inner points is given in Table III. For inner points the proposed approach yields in average 17\% improvement in comparison to the contour-based approach [25], 58\% in comparison to the contour-based approach [23] and $103 \%$ improvement in comparison to the intensity-based method [9]. The most significant improvement of the proposed approach in comparison to the previously introduced algorithms was shown for the periphery of the nucleus. The registration error for the end points for the proposed approach was $41 \%$ less than for the contour-based approach [25], $104 \%$ less than for the intensity based approach [9] and $185 \%$ less than the contour-based approach [23]. However in Seq. B4 the proposed approach performs $17 \%$ worse for end points compared to the contour-based method [25].

To demonstrate the dependence of the registration error on time we show the registration error averaged over keypoints tracked by the annotator $A_{2}$ for Seq. A2 (Fig. 6). It can be seen 


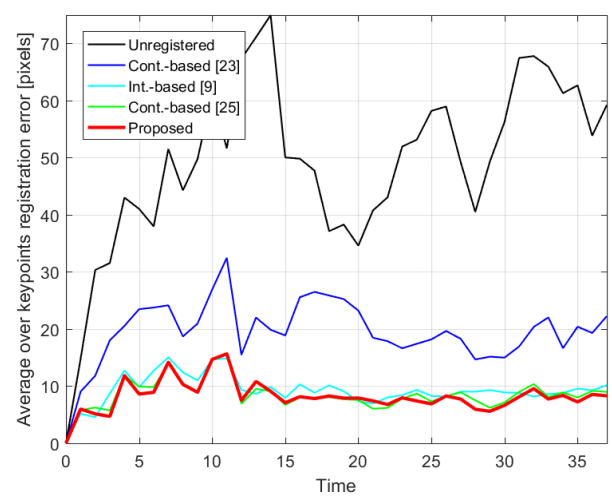

Fig. 6. Average registration error for the structures manually tracked by annotator $A_{1}$ in the image sequence Seq. A2 as a function of time.

that both elasticity-based approaches have similar behavior and yield better results than the other methods almost through the whole sequence. However the proposed approach performs slightly better in the beginning (time points 1-6) and in the end of the sequence (starting from time point 23 ). It can also be seen that the error fluctuations of the registration error for the contour-based approach [25], intensity-based approach [9] and the proposed approach is significantly less than the fluctuations for the contour-based approach [23]. We also show the registration error averaged over line features for Seq. B3 in Fig. 7. Here, the registration error of the proposed approach is the lowest almost through the whole sequence and the behavior of the registration error for all contour-based approaches is similar to Seq. A2. The registration error of the intensity-based approach is higher than in Seq. A2 due to intensity changes. However the error for the proposed approach and the contourbased approach [25] slightly increases starting from time point 25 while the increase rate for the intensity-based approach is much less.

To visualize the registration error we showed the tracks of the line features overlaid over the first frame of the Seq. B2 for unregistered data and after registration with different approaches. For better visual perception the line features are represented by points sampled with 30 pixel interval. It can be observed that the tracks of the points after registration with both elasticity based approaches are much more compact than after the registration with the contour-based approach [23] and intensity-based approach [9] indicating the lower registration error. Moreover, the tracks obtained after the registration using the proposed approach are slightly more compact than after registration using the previous elasticity-based approach [25] (the particular tracks are marked with white arrows).

\section{Method Parameters Study}

We have also studied the dependence of the registration results on the choice of method parameters. As it is described in section II the proposed approach have three parameters: the Poisson's ratio $\nu$, the Young's modulus $E$ and the target compliance $\gamma$. We used Seq. A2, A3 and B2 to study the influence of the parameters on the registration error for

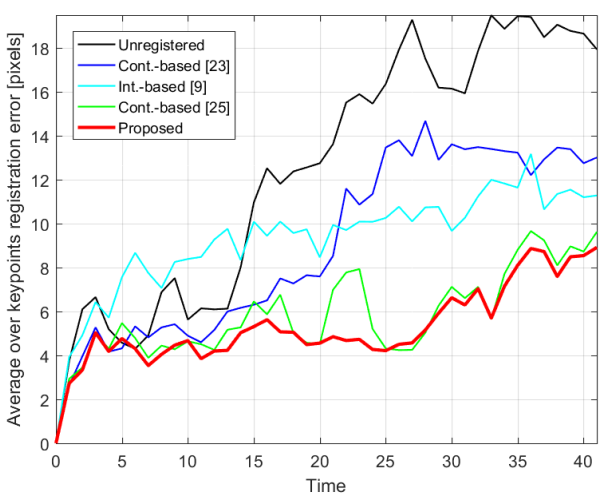

Fig. 7. Average registration error for the line features in the image sequence Seq. B3 as a function of time.

different phenotypes (Seq. A2 representing U2OS cell in interphase, Seq. A3 representing U2OS cell in mitosis, Seq. B2 representing HeLa cell in interphase).

First, we examined the influence of the Poisson's ratio on the registration results. We performed the computations of $\bar{e}_{\text {mean }}$ for the fixed values of Young's modulus and target compliance $(E=10 k P a, \gamma=0.5)$ and the value of $\nu \in[0.38,0.49]$ with the step size 0.01. The range of the tested values of $\nu$ was selected according to the latest studies on the Poisson's ratio value for cell nucleus [35], [38]. For Seq. A2 we took the registration error $\bar{e}_{\text {mean }}$ averaged over annotators, for Seq. B2 we computed the registration error $\bar{e}_{\text {mean }}$ for line features. The standard deviation of the registration error $\bar{e}_{\text {mean }}$ for Seq. A2, Seq. A3 and Seq. B2 were 0.008, 0.011 and 0.007 pixels respectively which is less than $0.2 \%$ in relation to the absolute values of the registration error. Thus the value of Poisson's ratio does not significantly influence the registration results if chosen from the range reported in previous work.

Next, we studied the behavior of the registration error depending on the values of $E$ and $\gamma$ for the fixed value of $\nu=0.4$. We computed the registration error $\bar{e}_{\text {mean }}$ of the proposed approach for the fixed value of $\nu$ and every pair of $E$ and $\gamma$ which values were chosen from the selected ranges. Recent studies show that the Young's modulus of the cell nucleus vary from 1 to $10 \mathrm{kPa}$ [36], [39]. However we extended the range of $E$ for our evaluations and took the values from the interval 0,5 to $15 \mathrm{kPa}$ with the step of $1 \mathrm{kPa}$. The target compliance regulates the stiffness of the contour matching process by enabling the purely geometrical target object to move to improve the numerical stability of dynamic integration. Namely, the target compliance allows the contour points of the target nucleus to slide along its tangent during the simulation process. Since $\gamma$ does not have a physical meaning for our evaluation we took $\gamma \in[0,6]$ with the step of 0.5 . The standard deviation of averaged over annotators registration error $\bar{e}_{\text {mean }}$ for Seq. A2 was 0.033 pixels. The standard deviation of the registration error $\bar{e}_{\text {mean }}$ for Seq. A3 was 0.011 pixels. And the standard deviation of the registration error computed for line features for Seq. B2 was 0.083 pixels. In Fig. 8 the mean registration error depending on $E$ and $\gamma$ and the histograms of mean registration error are shown. One can 

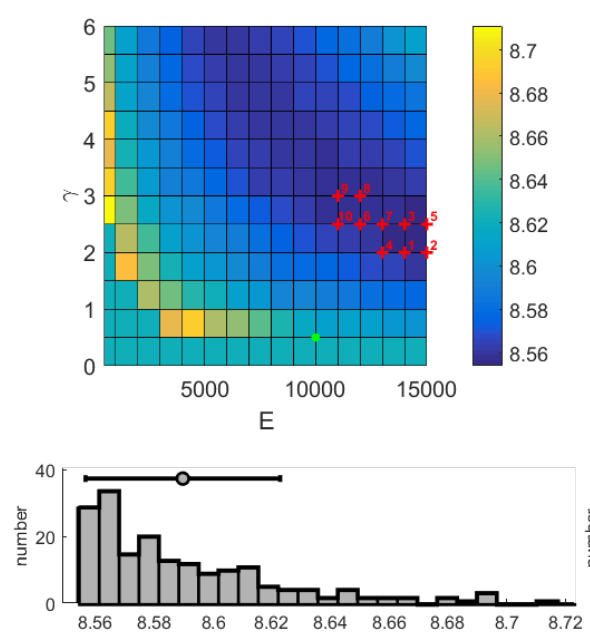

(a)
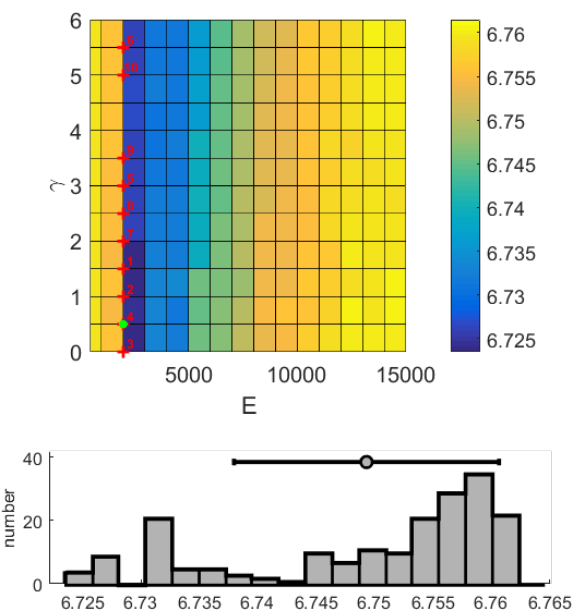

(b)
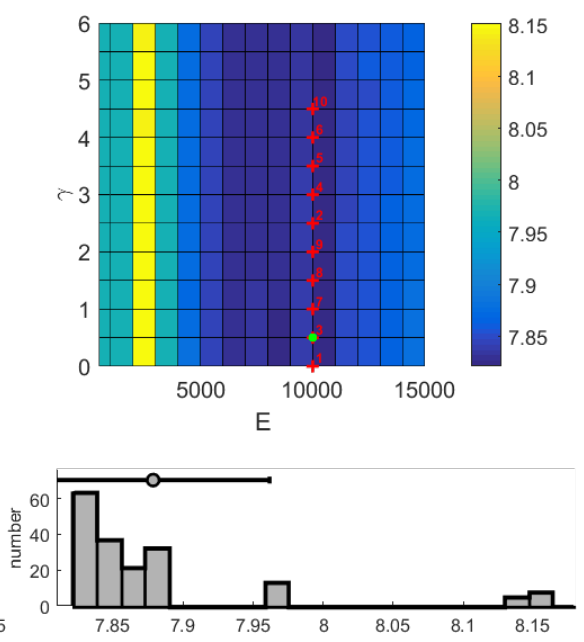

(c)

Fig. 8. The mean registration error of the proposed approach depending on $E$ and $\gamma$ and the histograms of mean registration error for different pairs of $E$ - $\gamma$ parameters and fixed value of Poisson's ration $\nu=0.4$. In (a) the mean registration error averaged over three annotators for Seq. A2 is shown, in (b) the mean registration error for Seq. A3 is shown, in (c) the mean registration error for line features for Seq. B2 is shown. The red crosses represent the parameter pairs for which the 10 lowest mean registration errors were obtained. The green dot shows the theoretical values of $E$ and $\gamma$ taken for the computation of results in Tables II and III.

see that for Seq. A3 and Seq. B2 the registration error does not significantly depend on $\gamma$ but depends on $E$. However the theoretical values of $E$ and $\gamma$ (plotted as green dot) taken for the evaluation results presented in Table II and III are within 10 optimal $E-\gamma$ pairs resulting the lowest registration errors (plotted as red crosses). For Seq. A2 one can see the inverse relationship between the optimal values of $E$ and $\gamma$. The optimal $E-\gamma$ pairs resulting 10 lowest registration errors are within $E \in[11,15] \mathrm{kPa}$ and $\gamma \in[2,3]$ which is higher than the theoretical range $1-10 \mathrm{kPa}$ [36]. However the difference between the registration error obtained for $E=10 \mathrm{kPa}$ and $\gamma=0.5$ from the minimal registration error obtained for $E=14 \mathrm{kPa}$ and $\gamma=2$ is just 0.06 pixels.

\section{Discussion}

We performed the evaluation of the proposed registration approach on two real microscopy image datasets specifically designed for cell image registration evaluation. It turned out that the proposed approach outperforms previous contourbased methods. The reason for that is the realistic dynamic elasticity model used to simulate the nucleus motion based on the motion of its contours. While in the previously introduced approach [23] any physical model was not employed and in our previously introduced contour-based approach [25] the less accurate elastostatic model was used.

The proposed approach also outperforms pairwise intensitybased registration approach for 6 out of 7 testing sequences. Generally speaking, intensity-based approaches are more beneficial than contour-based approaches as they use the information from entire image while contour-based approaches use only the information about the motion of cell contours. However intensity-based approaches usually does not imply any physical model of motion and rely only on the information taken directly from the images. This can cause problems if the images have high level of noise or significant structural changes in intensity during cell motion. Another limitation of intensity-based approaches is that they require some stable structures to be present in the images to be registered. These structures should have very limited local motion and mostly represent the motion and deformation of the cell. In order to fulfill this requirement the images are usually acquired in two channels where one channel represents the nucleus and another channel represents the particles of interest. Then the intensity-based registration approach is applied to the nucleus channel and the resulting deformation fields are used to compensate the motion of the nucleus in the particle channel [9]. However in some cases the image sequence can not be acquired in two channels and both nucleus and particles are shown in the same channel. In this case the application of intensity-based approach can influence the local motion of the particles which is usually the main biological interest in this type of applications. Taking this into account, the contourbased approaches can be beneficial and more universal for data acquired in one channel even though in some cases the registration accuracy of contour-based methods are lower than the accuracy of the intensity-based methods.

In was shown that the proposed approach is quite robust to the choice of method parameter. Two out of three method parameters (Poisson's ratio and Young's modulus) have clear physical meaning and can be chosen based on the theoretical values reported in the literature. Even though there is still no consensus on the exact values of these parameters for different cell phenotypes we have showed that variation of $E$ and $\nu$ in the range reported in literature $(E \in[1,10] \mathrm{kPa}$ and $\nu \in[0.38,0.48])$ do not lead to significant difference in registration results. The third parameter of the method (target object complience) also does not significantly influence the registration quality and can be chosen empirically.

Unlike the approach in [25] the proposed approach does not require separate rigid alignment step. Thanks to the model 
where the contour matching is embedded as constraints into the system of equation the proposed approach is capable to compensate for large displacements and rotation of the cell. In addition the solution is based on FE method and consists of three main steps which require solving the linear system of equations, setting the constraints by iterative closest point method and resolving the constraints by Gauss-Seidel method. It makes the calculations computationally efficient. We used SOFA framework to compute the simulation [40] and Matlab to compute the deformation fields. We determined the computation time for Seq. A2 containing 38 frames of $512 \times 512$ pixels on Windows workstation with an Intel Core 2 Quad CPU (2.4 GHz). The computation time was 3 min 46 sec. Note, that our implementation is not optimized and the computation time can be further reduced.

\section{CONCLUSION}

We have presented a contour-based approach for temporal non-rigid registration of cell nuclei in 2D live cell microscopy images. A quantitative experimental evaluation of the approach using real live cell microscopy data has been performed. We also investigated the influence of method parameters on registration results. The presented approach exploits forward nucleus motion simulation computed based on FE method. The resulting motion model defined on the set of triangulations allows computing the deformation fields to perform the image registration. By using dynamic elasticity model with contour matching embedded into the system of equation as constraints the proposed approach outperforms the existing contour-based approaches for all tested image sequences and pairwise intensity-based approach for all except one tested image sequences. Thanks to FE method used for calculations the proposed approach is computationally effective. In comparison to the existing contour-based approaches our approach can be straightforwardly extended to 3D by choosing the appropriate finite elements which is a topic for future work. In addition, the proposed solution scheme allows embedding some intensity information to the elastic deformation model by including additional constraints to the equations which is also planned for future work.

\section{APPENDIX A}

The stiffness matrix $\mathbf{K}$ is constructed by summing up the local stiffness matrices $\mathbf{K}_{e}$ for for every element $e \in \mathcal{E}_{k}$ of the triangulation $\mathcal{T}_{k}$ (this process is called the assembly [41]). The local stiffness matrix for $e$ element is defined as

$$
\mathbf{K}_{e}=\mathbf{R}_{e}^{T} \mathbf{G}_{e}^{T} \mathbf{D}_{e} \mathbf{G}_{e} \mathbf{R}_{e},
$$

where $\mathbf{G}_{e}$ is the strain-displacement matrix, $\mathbf{D}_{e}$ is the stressstrain matrix and $\mathbf{R}_{e}$ is a rotation matrix from corrotational formulation. The matrix $\mathbf{D}_{e}$ is defined as

$$
\mathbf{D}_{e}=\left[\begin{array}{ccc}
\lambda+2 \mu & \lambda & 0 \\
\lambda & \lambda+2 \mu & 0 \\
0 & 0 & \mu
\end{array}\right]
$$

where $\lambda$ and $\mu$ are the Lamé coefficients. The matrix $\mathbf{G}_{e}^{T}$ is defined as

$$
\mathbf{G}_{e}=[\ldots]
$$

and we refer the reader to [29] for the definition of the matrix $\mathbf{R}_{e}$.

The damping matrix $\mathbf{B}$ is defined as

$$
\mathbf{B}=\alpha \mathbf{K}+\beta \mathbf{M}
$$

where $\alpha$ and $\beta$ are the Rayleigh coefficients (in our approach we use $\alpha=10$ and $\beta=$ ?), $\mathbf{M}$ is the nucleus mass matrix and $\mathbf{K}$ is the stiffness matrix.

\section{ACKNOWLEDGMENT}

This work was supported by the Czech Science Foundation project 302/12/G157 and (ADD YOURS) .

\section{REFERENCES}

[1] C. A. Wilson and J. A. Theriot, "A correlation-based approach to calculate rotation and translation of moving cells," Image Processing, IEEE Transactions on, vol. 15, no. 7, pp. 1939-1951, 2006.

[2] A. P. Goobic, J. Tang, and S. T. Acton, "Image stabilization and registration for tracking cells in the microvasculature," Biomedical Engineering, IEEE Transactions on, vol. 52, no. 2, pp. 287-299, 2005.

[3] S. Baheerathan, F. Albregtsen, and H. Danielsen, "Registration of serial sections of mouse liver cell nuclei," Journal of microscopy, vol. 192, no. 1, pp. 37-53, 1998.

[4] B. Rieger, C. Molenaar, R. Dirks, and L. Van Vliet, "Alignment of the cell nucleus from labeled proteins only for $4 \mathrm{~d}$ in vivo imaging," Microscopy research and technique, vol. 64, no. 2, pp. 142-150, 2004.

[5] M. van de Giessen, A. van der Laan, E. A. Hendriks, M. Vidorreta, J. H. Reiber, C. R. Jost, H. J. Tanke, and B. P. Lelieveldt, "Fully automated attenuation measurement and motion correction in flip image sequences," Medical Imaging, IEEE Transactions on, vol. 31, no. 2, pp. 461-473, 2012.

[6] S.-e.-A. Raza, A. Humayun, S. Abouna, T. W. Nattkemper, D. B. A. Epstein, M. Khan, and N. M. Rajpoot, "Ramtab: Robust alignment of multi-tag bioimages," PLoS ONE, vol. 7, no. 2, p. e30894, 022012.

[7] S. Ozere, P. Bouthemy, F. Spindler, P. Paul-Gilloteaux, and C. Kervrann, "Robust parametric stabilization of moving cells with intensity correction in light microscopy image sequences," in Proc. 10th IEEE International Symposium on Biomedical Imaging: Nano to Macro, 2013, pp. 468-471.

[8] J. Mattes, J. Nawroth, P. Boukamp, R. Eils, and K. M. Greulich-Bode, "Analyzing motion and deformation of the cell nucleus for studying co-localizations of nuclear structures," in Proc. 3rd IEEE International Symposium on Biomedical Imaging: Nano to Macro, 2006, pp. 1044 1047.

[9] I.-H. Kim, Y.-C. M. Chen, D. L. Spector, R. Eils, and K. Rohr, "Nonrigid registration of 2-d and 3-d dynamic cell nuclei images for improved classification of subcellular particle motion," IEEE Transactions on Image Processing, vol. 20, no. 4, pp. 1011-1022, 2011.

[10] M. Tektonidis, I.-H. Kim, Y.-C. M. Chen, R. Eils, D. L. Spector, and K. Rohr, "Non-rigid multi-frame registration of cell nuclei in live cell fluorescence microscopy image data," Medical image analysis, vol. 19, no. 1, pp. 1-14, 2015.

[11] C. Medioni, F. Besse, X. Descombes, and G. Malandarne, "Motion compensation in two-photon microscopy temporal series," in 2015 IEEE 12th International Symposium on Biomedical Imaging (ISBI). IEEE, apr 2015, pp. 1584-1587.

[12] T. Du and M. Wasser, "3d image stack reconstruction in live cell microscopy of drosophila muscles and its validation," Cytometry Part A, vol. 75, no. 4, pp. 329-343, 2009.

[13] L. Hogrebe, A. R. Paiva, E. Jurrus, C. Christensen, M. Bridge, L. Dai, R. L. Pfeiffer, P. R. Hof, B. Roysam, J. R. Korenberg et al., "Serial section registration of axonal confocal microscopy datasets for longrange neural circuit reconstruction," Journal of neuroscience methods, vol. 207, no. 2, pp. 200-210, 2012.

[14] K. S. Lorenz, P. Salama, K. W. Dunn, and E. J. Delp, "A multi-resolution approach to non-rigid registration of microscopy images," in Biomedical Imaging (ISBI), 2012 9th IEEE International Symposium on. IEEE, 2012, pp. 198-201.

[15] S. Saalfeld, R. Fetter, A. Cardona, and P. Tomancak, "Elastic volume reconstruction from series of ultra-thin microscopy sections," Nature Methods, vol. 9, no. 7, pp. 717-720, 2012. 
[16] A. Akselrod-Ballin, D. Bock, R. C. Reid, and S. K. Warfield, "Accelerating image registration with the johnson-lindenstrauss lemma: Application to imaging 3-d neural ultrastructure with electron microscopy," Medical Imaging, IEEE Transactions on, vol. 30, no. 7, pp. 1427-1438, 2011.

[17] R. Tomer, A. S. Denes, K. Tessmar-Raible, and D. Arendt, "Profiling by image registration reveals common origin of annelid mushroom bodies and vertebrate pallium," Cell, vol. 142, no. 5, pp. 800-809, 2010.

[18] H. Peng, P. Chung, F. Long, L. Qu, A. Jenett, A. M. Seeds, E. W. Myers, and J. H. Simpson, "Brainaligner: $3 \mathrm{~d}$ registration atlases of drosophila brains," nAture methods, vol. 8, no. 6, pp. 493-498, 2011.

[19] D. Sage, F. R. Neumann, F. Hediger, S. M. Gasser, and M. Unser, "Automatic tracking of individual fluorescence particles: application to the study of chromosome dynamics." IEEE transactions on image processing : a publication of the IEEE Signal Processing Society, vol. 14, no. 9, pp. 1372-83, sep 2005.

[20] P. Matula, P. Matula, M. Kozubek, and V. Dvořák, "Fast point-based 3-d alignment of live cells," IEEE Transactions on Image Processing, vol. 15, no. 8, pp. 2388-2396, 2006.

[21] O. Dzyubachyk, J. Essers, W. a. van Cappellen, C. Baldeyron, A. Inagaki, W. J. Niessen, and E. Meijering, "Automated analysis of time-lapse fluorescence microscopy images: from live cell images to intracellular foci." Bioinformatics (Oxford, England), vol. 26, no. 19, pp. 2424-30, oct 2010.

[22] S. Yang, D. Kohler, K. Teller, T. Cremer, P. Le Baccon, E. Heard, R. Eils, and K. Rohr, "Nonrigid registration of 3-d multichannel microscopy images of cell nuclei." IEEE transactions on image processing, vol. 17, no. 4, pp. 493-9, apr 2008.

[23] J. De Vylder, W. H. De Vos, E. M. Manders, and W. Philips, "2d mapping of strongly deformable cell nuclei-based on contour matching," Cytometry Part A, vol. 79, no. 7, pp. 580-588, 2011.

[24] E. Gladilin, S. Goetze, J. MATEOS-LANGERAK, R. Van Driel, R. Eils, and K. Rohr, "Shape normalization of $3 \mathrm{~d}$ cell nuclei using elastic spherical mapping," Journal of microscopy, vol. 231, no. 1, pp. 105114, 2008.

[25] D. V. Sorokin, M. Tektonidis, K. Rohr, and P. Matula, "Non-rigid contour-based temporal registration of $2 \mathrm{D}$ cell nuclei images using the Navier equation," in 2014 IEEE 11th International Symposium on Biomedical Imaging (ISBI). IEEE, apr 2014, pp. 746-749.

[26] D. V. Sorokin, J. Suchánková, E. Bártová, and P. Matula, "Visualizing stable features in live cell nucleus for evaluation of the cell global motion compensation," Folia biologica, vol. 60, pp. 45-9, 2014.

[27] Y. Tseng, J. S. Lee, T. P. Kole, I. Jiang, and D. Wirtz, "Microorganization and visco-elasticity of the interphase nucleus revealed by particle nanotracking," Journal of Cell Science, vol. 117, no. 10, pp. 2159-2167, 2004.

[28] M. H. Sadd, Elasticity: Theory, Applications, and Numerics, 2nd ed Elsevier, 2009.

[29] M. Nesme, Y. Payan, and F. Faure, "Efficient, Physically Plausible Finite Elements," in Eurographics, ser. Short papers, Dublin, Ireland, Aug. 2005.

[30] C. A. Felippa, "A study of optimal membrane triangles with drilling freedoms," Computer Methods in Applied Mechanics and Engineering, vol. 192, no. 1618 , pp. 2125 - 2168, 2003.

[31] S. Rusinkiewicz and M. Levoy, "Efficient variants of the icp algorithm," in 3-D Digital Imaging and Modeling, 2001. Proceedings. Third International Conference on. IEEE, 2001, pp. 145-152.

[32] H. Courtecuisse, J. Allard, P. Kerfriden, S. P. Bordas, S. Cotin, and C. Duriez, "Real-time simulation of contact and cutting of heterogeneous soft-tissues," Medical image analysis, vol. 18, no. 2, pp. 394-410, 2014.

[33] C. Duriez, F. Dubois, A. Kheddar, and C. Andriot, "Realistic haptic rendering of interacting deformable objects in virtual environments," IEEE Transactions on Visualization and Computer Graphics, vol. 12, no. 1, pp. 36-47, 2006.

[34] A. Y. Kondrat'ev and D. V. Sorokin, "Automatic detection of laserinduced structures in live cell fluorescent microscopy images using snakes with geometric constraints," in 2016 24nd International Conference on Pattern Recognition (ICPR). IEEE, 2016, p. Accepted for publication.

[35] E. Gladilin, M. Schulz, C. Kappel, and R. Eils, "Contactless determination of nuclear compressibility using $3 \mathrm{~d}$ image-and model-based analysis of drug-induced cellular deformation," Journal of microscopy, vol. 240, no. 3, pp. 216-226, 2010.

[36] J. Lammerding, "Mechanics of the nucleus," Comprehensive Physiology, 2011.
[37] B. Houchmandzadeh, J. F. Marko, D. Chatenay, and A. Libchaber, "Elasticity and structure of eukaryote chromosomes studied by micromanipulation and micropipette aspiration," The Journal of cell biology, vol. 139, no. 1, pp. 1-12, 1997.

[38] P. G. Avalos, M. Reichenzeller, R. Eils, and E. Gladilin, "Probing compressibility of the nuclear interior in wild-type and lamin deficient cells using microscopic imaging and computational modeling," Journal of biomechanics, vol. 44, no. 15, pp. 2642-2648, 2011.

[39] N. Caille, O. Thoumine, Y. Tardy, and J.-J. Meister, "Contribution of the nucleus to the mechanical properties of endothelial cells," Journal of biomechanics, vol. 35, no. 2, pp. 177-187, 2002.

[40] J. Allard, S. Cotin, F. Faure, P.-J. Bensoussan, F. Poyer, C. Duriez, H. Delingette, and L. Grisoni, "Sofa-an open source framework for medical simulation," in MMVR 15-Medicine Meets Virtual Reality, vol. 125. IOP Press, 2007, pp. 13-18.

[41] D. Baraff and A. Witkin, "Large steps in cloth simulation," in Proceedings of the 25th annual conference on Computer graphics and interactive techniques. ACM, 1998, pp. 43-54.

[42] G. Nikishkov, "Introduction to the finite element method," University of Aizu, 2004.

[43] M. Müller and M. Gross, "Interactive virtual materials," in Proceedings of Graphics Interface 2004. Canadian Human-Computer Communications Society, 2004, pp. 239-246. 